\title{
Principles of Discrete Time Mechanics: II. Classical Field Theory
}

\author{
George Jaroszkiewicz and Keith Norton \\ Department of Mathematics, University of Nottingham \\ University Park, Nottingham NG7 2RD, UK
}

$19^{\text {th }}$ December 1996

\begin{abstract}
We apply the principles discussed in an earlier paper to the construction of discrete time field theories. We derive the discrete time field equations of motion and Noether's theorem and apply them to the Schrödinger equation to illustrate the methodology. Stationary solutions to the discrete time Schrödinger wave equation are found to be identical to standard energy eigenvalue solutions except for a fundamental limit on the energy. Then we apply the formalism to the free neutral Klein Gordon system, deriving the equations of motion and conserved quantities such as the linear momentum and angular momentum. We show that there is an upper bound on the magnitude of linear momentum for physical particle-like solutions. We extend the formalism to the charged scalar field coupled to Maxwell's electrodynamics in a gauge invariant way. We apply the formalism to include the Maxwell and Dirac fields, setting the scene for second quantisation of discrete time mechanics and discrete time Quantum Electrodynamics.
\end{abstract}

\section{Introduction}

THIS paper follows an earlier paper on the principles of discrete time mechanics as formulated for point particle theories, hereafter referred to as Paper $I$ [1]. These principles are applied in this paper to systems described by fields $\varphi^{\alpha}(t, \mathbf{x})$ in one time and 3 spatial dimensions, where $\alpha$ denotes spin component and field types. Units will normally be taken to give $c=\hbar=1$. The summation convention will apply only to repeated small Greek indices except where otherwise stated.

In discrete time mechanics dynamical variables take on values at times $t_{n}, n=$ $0,1,2, \ldots N$, with the discrete time intervals $\Delta t_{n} \equiv t_{n+1}-t_{n}, n=0,1,2, \ldots$ given by $\Delta t_{n}=T$, where $T>0$ is the fundamental time unit of the system. An important principle of our formulation is that the mechanics is regarded as exact regardless of the magnitude of $T$, and not an exercise in approximation. This means that invariants of the motion are constructed to be conserved precisely and not up to some given order in $T$. 
As in Paper I our discrete time approach takes its cues from continuous time mechanics, but ultimately is more than an approximation to such a formalism. In general the continuous time Lagrangians $L$ for the systems of interest to us in this paper are the spatial integrals of Lagrange densities $\mathcal{L}$ of the form $\mathcal{L}=\mathcal{L}\left(\boldsymbol{\varphi}, \partial_{t} \boldsymbol{\varphi}, \nabla \boldsymbol{\varphi}\right)$.

Important concepts in our methodology are those of the system function and virtual path. In our formalism the system function has the status occupied by the Lagrangian in continuous time mechanics. We may calculate equations of motion and conserved quantities if we know the system function. We may also use the system function to define conjugate momenta and to quantise our theory. We construct the system function from the continuous time Lagrangian by integrating over certain trajectories, which we refer to as virtual paths. These are not solutions to the normal Euler-Lagrange equations of motion but paths chosen according to specific criteria such as gauge invariance.

In our approach we shall discuss discrete time mechanics based on a temporal lattice with successive points given by

$$
t_{n+1}-t_{n}=T
$$

where $T$ is non-zero and independent of $n$. If $\varphi(t)$ is a dynamical variable depending on time we will normally define a virtual path $\tilde{\varphi}$ from $\varphi_{n} \equiv \varphi\left(t_{n}\right)$ to $\varphi_{n+1} \equiv \varphi\left(t_{n+1}\right)$ in $\varphi$-value space by the linear rule

$$
\tilde{\varphi}(t) \equiv \lambda \varphi_{n+1}+\bar{\lambda} \varphi_{n}
$$

where $\lambda$ runs from 0 to 1 and $\bar{\lambda} \equiv 1-\lambda$. This means that over the interval $\left[t_{n}, t_{n+1}\right]$ the time $t$ is related to $\lambda$ by the rule

$$
t=t_{n}+\lambda T=\lambda t_{n+1}+\bar{\lambda} t_{n}
$$

This prescription is modified when we include gauge invariance, as discussed in $\S 8$. With the above virtual path prescription time derivatives are naturally turned into differences, i.e.

$$
\frac{\partial}{\partial t} \tilde{\varphi}(t)=\frac{\varphi_{n+1}-\varphi_{n}}{T} .
$$

It is possible to use a different temporal lattice. A popular choice in recent years has been the so-called $q$-lattice, where we define $t_{n+1} \equiv q t_{n}$, where $q$ is real and not equal to unity. Then we would define $\varphi_{n} \equiv \varphi\left(t_{n}\right)=\varphi\left(q^{n} t_{0}\right), t_{0} \neq 0$. The virtual path prescription on the interval $\left[t_{n}, t_{n+1}\right]$ would be the same as (3) with

$$
t=t_{n}+\lambda\left(t_{n+1}-t_{n}\right)=\lambda t_{n+1}+\bar{\lambda} t_{n}
$$

and so in the interval $\left[t_{n}, t_{n+1}\right]$ the temporal derivative would be replaced by the so-called $q$-derivative

$$
\frac{\partial}{\partial t} \tilde{\varphi}(t)=\frac{\varphi_{n+1}-\varphi_{n}}{t_{n+1}-t_{n}}=\frac{\varphi\left(q t_{n}\right)-\varphi\left(t_{n}\right)}{(q-1) t_{n}} .
$$


We may go further and accommodate the so-called symmetric derivative [2] given by

$$
\frac{\bar{\partial}}{\partial t} \tilde{\varphi}(t) \equiv \frac{\varphi\left(q t_{n}\right)-\varphi\left(q^{-1} t_{n}\right)}{\left(q-q^{-1}\right) t_{n}},
$$

but in this particular case the virtual temporal path (viewed in terms of successive intervals) is not continuous everywhere. We may even generalise in the manner of Klimek [2] and define $t_{n+1} \equiv \phi\left(t_{n}\right)$, where $\phi$ is some chosen real valued function and then the above prescription gives

$$
\frac{\partial}{\partial t} \tilde{\varphi}(t)=\frac{\varphi\left(\phi\left(t_{n}\right)\right)-\varphi\left(t_{n}\right)}{\phi\left(t_{n}\right)-t_{n}} .
$$

The regular temporal lattice is given by the function $\phi(t)=t+T$ in Klimek's formulation. We shall use this lattice throughout this paper, applying it to the Schrödinger wave equation, the free neutral scalar field, the free charged scalar field, the Maxwell fields and potentials and to the Dirac field, and show how to couple these fields in a gauge invariant way. The quantisation of our discrete time field theories will be discussed in the next paper of this series, Paper III, following the principles discussed in Paper I. A detailed discussion of quantum electrodynamics will be presented in Paper $I V$ of this series.

The plan of this paper is as follows. First we review Hamilton's principal function in continuous time field theory and then discuss the related concept of system function in discrete time field theory. We derive the field Cadzow's equations of motion and discuss the Maeda-Noether theorem for the construction of invariants of the motion. Then we apply our formalism to the Schrödinger equation, as an example of a $\mathcal{D} \mathcal{Q}$ process, where $\mathcal{Q}$ represents the process of quantisation and $\mathcal{D}$ represents the process of discretisation of time. The resulting theory is a classical field theory which is not the same as the result of the $\mathcal{Q D}$ process discussed in Paper I.

Then we turn to relativistic theories. The $\mathcal{D}$ process breaks Lorentz invariance, but in a less emphatic fashion than space-time lattice theories such as those discussed by Yamamoto et al. [3, [4]. The systems we study here are the free neutral and charged Klein-Gordon fields, the Maxwell potentials and their coupling to the charged scalar field, and the Dirac equation. This sets the scene for second quantisation, i.e. the $\mathcal{Q D} \mathcal{Q}$ process, which is discussed in Paper III.

\section{Hamilton's principal function}

In this section we review some aspects of continuous time mechanics field theory which are relevant to our method of discretisation. Following the principles outlined in Paper I we shall consider a dynamical field system for which we have knowledge of the dynamical variables $\varphi^{\alpha}$ at a sequence of times $t_{n}, n=0,1,2, \ldots N$, where $t_{n+1} \equiv t_{n}+$ $T$. An important construction in continuous time mechanics is Hamilton's principal function $S^{n}(T)$, defined as the integral

$$
S^{n}(T) \equiv \int_{t_{n}}^{t_{n+1}} d t \int d^{3} \mathbf{x} \mathcal{L}
$$


over the classical solution to the field equations which satisfies the boundary conditions

$$
\varphi_{n}^{\alpha}(\mathbf{x}) \equiv \varphi^{\alpha}\left(t_{n}, \mathbf{x}\right), \quad \varphi_{n+1}^{\alpha}(\mathbf{x}) \equiv \varphi^{\alpha}\left(t_{n+1}, \mathbf{x}\right) .
$$

$S^{n}(T)$ is a functional of $\boldsymbol{\varphi}_{n}$ and $\boldsymbol{\varphi}_{n+1}$ and is the continuous time analogue of the system function in discrete time mechanics. If the momentum $\pi^{\alpha}$ conjugate to $\varphi^{\alpha}$ is defined by $\pi^{\alpha} \equiv \frac{\partial \mathcal{L}}{\partial \dot{\varphi}^{\alpha}}$ and the Hamiltonian $H$ defined by

$$
H \equiv \int d^{3} \mathbf{x}\left\{\pi^{\alpha} \dot{\varphi}^{\alpha}-\mathcal{L}\right\}
$$

then for an infinitesimal variation $\delta \varphi^{\alpha}$ of the fields we find

$$
\delta S^{n}(T) \underset{c}{=}\left[-\delta t H+\int d^{3} \mathbf{x} \pi^{\alpha} \delta \varphi^{\alpha}\right]_{t_{n}}^{t_{n+1}}
$$

where the symbol $\underset{c}{=}$ denotes an equality holding over the true or dynamical trajectory. From this we deduce the functional derivatives

$$
\frac{\delta S^{n}}{\delta \varphi_{n}^{\alpha}(\mathbf{x})}=-\pi_{n}^{\alpha}(\mathbf{x}), \quad \frac{\delta S^{n}}{\delta \varphi_{n+1}^{\alpha}(\mathbf{x})}=\pi_{n+1}^{\alpha}(\mathbf{x})
$$

and the partial derivatives

$$
\frac{\partial S^{n}}{\partial t_{n}}=H^{n}, \quad \frac{\partial S^{n}}{\partial t_{n+1}}=-H^{n+1},
$$

where $H^{n}$ is the value of the Hamiltonian at time $t_{n}$.

By the process of constructing $S^{n}$ for each of the intervals $\left[t_{n}, t_{n+1}\right], n=0,1, \ldots, N-$ 1 , we arrive at an action integral from $t_{0}$ to $t_{N}$ which is equivalent to the action sum

$$
A^{N} \equiv \sum_{n=0}^{N-1} S^{n}
$$

If the end point field values are held fixed (at times $t_{0}, t_{N}$ ) then this sum depends on the field values $\varphi_{n}^{\alpha}(\mathbf{x})$ at the intermediate time $t_{n}, n=1,2, \ldots, N-1$. If now we vary these intermediate fields and apply a variational principle we find the equations

$$
\frac{\delta}{\delta \varphi_{n}^{\alpha}(\mathbf{x})}\left\{S^{n-1}+S^{n}\right\} \underset{c}{=} 0, \quad 0<n<N
$$

These are the continuous time mechanics analogues of Cadzow's equations of motion [5] in discrete time field theory, discussed in the next section. The above equations (16) essentially ensure the continuity of the conjugate momenta at the end points of the sub-intervals. 


\section{Discrete time field theory}

We turn now to the discretisation of Lagrange based field theories based on the construction of the system function $F^{n}$ using the principles outlined in Paper $I$. By definition, the system function is the integral

$$
F^{n}=\int_{t_{n}}^{t_{n+1}} d t \int d^{3} \mathbf{x} \mathcal{L}\left(\tilde{\boldsymbol{\varphi}}, \partial_{t} \tilde{\boldsymbol{\varphi}}, \nabla \tilde{\boldsymbol{\varphi}}\right)
$$

with some choice of virtual path $\tilde{\varphi}$ in field value space. Once the temporal integral has been carried out the system function is a function of the field values $\varphi_{n}^{\alpha}(\mathbf{x}) \equiv \varphi^{\alpha}\left(t_{n}, \mathbf{x}\right)$ at times $t_{n}, t_{n+1}$ and their spatial derivatives at those times.

Given the system function, the action sum $A^{N}$ is given by

$$
A^{N}=\sum_{n=0}^{N-1} F^{n}
$$

and it is this which replaces the action sum (15) in continuous time mechanics. We derive the equations of motion using (18) and a variational principle in the standard fashion [5]. Consider infinitesimal variations of the fields with fixed end-points, $\delta \boldsymbol{\varphi}_{0}(\mathbf{x})=\delta \boldsymbol{\varphi}_{N}(\mathbf{x})=\mathbf{0}$. Assuming the fields fall off rapidly at spatial infinity, we apply Cadzow's action principle to obtain the discrete time field equations of motion

$$
\frac{\delta}{\delta \varphi_{n}^{\alpha}(\mathbf{x})}\left\{F^{n}+F^{n-1}\right\} \underset{c}{=} 0, \quad 0<n<N
$$

which are equivalent to

$$
\frac{\partial}{\partial \varphi_{n}^{\alpha}}\left\{\mathcal{F}^{n}+\mathcal{F}^{n-1}\right\} \underset{c}{=} \nabla \cdot \frac{\partial}{\partial \nabla \varphi_{n}^{\alpha}}\left\{\mathcal{F}^{n}+\mathcal{F}^{n-1}\right\},
$$

where $\mathcal{F}^{n} \equiv \mathcal{F}\left(\boldsymbol{\varphi}_{n}, \nabla \boldsymbol{\varphi}_{n}, \boldsymbol{\varphi}_{n+1}, \nabla \boldsymbol{\varphi}_{n+1}\right)$ is the system function density defined by

$$
\mathcal{F}^{n} \equiv \int_{t_{n}}^{t_{n+1}} d t \mathcal{L}\left(\tilde{\boldsymbol{\varphi}}, \partial_{t} \tilde{\boldsymbol{\varphi}}, \nabla \tilde{\boldsymbol{\varphi}}\right)
$$

The construction of constants of the motion is straightforward using Noether's theorem applied to discrete systems. Consider an infinitesimal transformation $\delta \boldsymbol{\varphi}_{n}, \delta \boldsymbol{\varphi}_{n+1}$ of the fields which leaves the system function unchanged. Then using the equation of motion we deduce that the quantity

$$
C^{n} \equiv \int d^{3} \mathbf{x}\left\{\frac{\partial \mathcal{F}^{n}}{\partial \varphi_{n}^{\alpha}}-\nabla \cdot \frac{\partial \mathcal{F}^{n}}{\partial \nabla \varphi_{n}^{\alpha}}\right\} \delta \varphi_{n}^{\alpha}
$$

is conserved on dynamical trajectories. In Paper $I$ we referred to this as the MaedaNoether theorem [6].

For linear momentum consider a transformation of the fields by the infinitesimal shift

$$
\varphi_{n}^{\alpha}(\mathbf{x}) \rightarrow \varphi_{n}^{\alpha \prime}(\mathbf{x})=\varphi_{n}^{\alpha}(\mathbf{x})+\delta \mathbf{a} \cdot \nabla \varphi_{n}^{\alpha}(\mathbf{x})
$$


Assuming that the Lagrange density is not explicitly dependent on position then we find that the conserved linear momentum $\mathbf{P}^{n}$ is given by

$$
\mathbf{P}^{n} \equiv \int d^{3} \mathbf{x}\left(\nabla \varphi_{n}^{\alpha}\right)\left\{\frac{\partial \mathcal{F}^{n}}{\partial \varphi_{n}^{\alpha}}-\nabla \cdot\left(\frac{\partial \mathcal{F}^{n}}{\partial \nabla \varphi_{n}^{\alpha}}\right)\right\} .
$$

For orbital angular momentum, consider a transformation of the fields by an infinitesimal rotation

$$
\varphi_{n}^{\alpha}(\mathbf{x}) \rightarrow \varphi_{n}^{\alpha \prime}(\mathbf{x})=\varphi_{n}^{\alpha}(\mathbf{x})+(\delta \boldsymbol{\omega} \times \mathbf{x}) \cdot \nabla \varphi_{n}^{\alpha}(\mathbf{x})+\delta \boldsymbol{\omega} \cdot \mathbf{s}^{\alpha \beta} \varphi_{n}^{\beta}(\mathbf{x}),
$$

where $\mathbf{s}^{\alpha \beta}$ is the transformation matrix for the spin indices. From this we may construct the conserved angular momentum

$$
\mathbf{L}^{n} \equiv \int d^{3} \mathbf{x}\left(\mathbf{x} \times \nabla \varphi_{n}^{\alpha}+\mathbf{s}^{\alpha \beta} \varphi_{n}^{\beta}\right)\left\{\frac{\partial \mathcal{F}^{n}}{\partial \varphi_{n}^{\alpha}}-\nabla \cdot\left(\frac{\partial \mathcal{F}^{n}}{\partial \nabla \varphi_{n}^{\alpha}}\right)\right\} .
$$

Other conserved quantities such as electric charge are just as readily obtained by the same method.

\section{The discrete time Schrödinger equation}

In Paper $I$ we discussed the possible non-commutativity of the processes of quantisation $\mathcal{Q}$ and discretisation $\mathcal{D}$. In that paper we presented an approach to $\mathcal{Q}$ after the application of $\mathcal{D}$ to point particle Lagrangians. Here we shall consider applying our $\mathcal{D}$ to a case where $\mathcal{Q}$ has already been carried out. The first application of our approach to the temporal discretisation of field theories is chosen to be the Schrödinger wave equation for a particle in an external time-independent potential.

First we note that the Schrödinger equation may be derived as a classical field equation in continuous time mechanics using the wave function $\Psi$ and its complex conjugate $\Psi^{*}$ as dynamical field variables with the Lagrangian density

$$
\mathcal{L}=\frac{1}{2} i \hbar\left(\Psi^{*} \partial_{t} \Psi-\partial_{t} \Psi^{*} \Psi\right)-\frac{\hbar^{2}}{2 m} \Psi^{*} \overleftarrow{\nabla} \cdot \vec{\nabla} \Psi-V(\mathbf{x}) \Psi^{*} \Psi
$$

The Euler-Lagrange equation of motion

$$
\partial_{t} \frac{\partial \mathcal{L}}{\partial \partial_{t} \Psi^{*}}+\nabla \cdot \frac{\partial \mathcal{L}}{\partial \nabla \Psi^{*}}=\frac{\partial \mathcal{L}}{\partial \Psi^{*}}
$$

leads to the usual Schrödinger equation

$$
i \hbar \partial_{t} \Psi \underset{c}{=}-\frac{\hbar^{2} \nabla^{2}}{2 m} \Psi+V(\mathbf{x}) \Psi
$$

and similarly for the complex conjugate wave-function.

Turning to our discretisation process, we define our virtual paths by

$$
\begin{aligned}
\tilde{\Psi}_{n}(\tilde{t}, \mathbf{x}) & \equiv \lambda \Psi_{n+1}(\mathbf{x})+\bar{\lambda} \Psi_{n}(\mathbf{x}), \\
\tilde{\Psi}_{n}^{*}(\tilde{t}, \mathbf{x}) & \equiv \lambda \Psi_{n+1}^{*}(\mathbf{x})+\bar{\lambda} \Psi_{n}^{*}(\mathbf{x}), \\
\tilde{t}(\lambda) & \equiv t_{n}+\lambda\left(t_{n+1}-t_{n}\right)
\end{aligned}
$$


for $\tilde{t} \in\left[t_{n}, t_{n+1}\right]$, where $\Psi_{n}(\mathbf{x}) \equiv \Psi\left(t_{n}, \mathbf{x}\right)$, etc. and $0 \leq \lambda \leq 1$. Then the system function turns out to be

$$
\begin{aligned}
\mathcal{F}^{n}= & \frac{i \hbar}{2}\left\{\Psi_{n}^{*} \Psi_{n+1}-\Psi_{n+1}^{*} \Psi_{n}\right\} \\
& -\frac{T}{6}\left\{2\left|\Psi_{n+1}\right|^{2}+2\left|\Psi_{n}\right|^{2}+\Psi_{n+1}^{*} \Psi_{n}+\Psi_{n}^{*} \Psi_{n+1}\right\} V(\mathbf{x}) \\
& -\frac{\hbar^{2} T}{12 m}\left\{2\left|\nabla \Psi_{n+1}\right|^{2}+2\left|\nabla \Psi_{n}\right|^{2}+\Psi_{n+1}^{*} \overleftarrow{\nabla} \cdot \vec{\nabla} \Psi_{n}+\Psi_{n}^{*} \overleftarrow{\nabla} \cdot \vec{\nabla} \Psi_{n+1}\right\} \cdot(31
\end{aligned}
$$

Using (31), Cadzow's equation of motion for $\Psi$ is

$$
i \hbar \frac{\left(\Psi_{n+1}-\Psi_{n-1}\right)}{2 T}=\left\{-\frac{\hbar^{2} \nabla^{2}}{2 m}+V(\mathbf{x})\right\} \frac{\left(\Psi_{n+1}+4 \Psi_{n}+\Psi_{n-1}\right)}{6}
$$

and similarly for the complex conjugate. This is our discretisation of the Schrödinger equation and represents the process $\mathcal{D} \mathcal{Q}$ applied to a standard continuous time mechanics point particle system.

Although in this theory there is no direct concept of a Hamiltonian, this does not mean that there are no conserved quantities analogous to continuous time energy. We define a stationary state solution to the discrete time Schrödinger equation (32) to be of the form

$$
\Psi_{n}(\mathbf{x}) \equiv e^{-i \epsilon n T / \hbar} \psi(\mathbf{x})
$$

where $\epsilon$ may be thought of as a discrete time energy. Then we find (32) reduces to

$$
E \psi(\mathbf{x})=\left\{-\frac{\hbar^{2} \nabla^{2}}{2 m}+V(\mathbf{x})\right\} \psi(\mathbf{x})
$$

where the eigenvalue $E$ is given by

$$
E=\frac{3 \hbar \sin (\epsilon T / \hbar)}{T\{\cos (\epsilon T / \hbar)+2\}}
$$

A plot of $y \equiv E T / \hbar$ as a function of $x \equiv \epsilon T / \hbar$ shows a periodic graph which passes through $\epsilon=0$ with a slope of unity, so that for low energies (compared to $\hbar / T) \epsilon$ is virtually identical with $E$. Then the graph rises to a local maximum when

$$
\epsilon=\frac{2 \pi \hbar}{3 T}
$$

at which point $E$ has the value $E_{\max }=\frac{\sqrt{3} \hbar}{T}$. If, as we imagine, $T$ is a very small timescale, such as the Planck time, then $E_{\max }$ is in practical terms far beyond any energy scale encountered in the laboratory or in any astrophysical or cosmological context, except possibly close to the Big Bang. Beyond the local maximum at $\epsilon=\frac{2 \pi \hbar}{3 T}$ the graph falls to zero and goes negative, followed by a local minimum and a return to zero. Thereafter it repeats this overall pattern. We note here the possibility of resonances, where the values of $\epsilon$ differ by multiples of $\frac{2 \pi \hbar}{T}$. These vanish in the limit $T \rightarrow 0$. 
Assuming $0<E<\frac{\sqrt{3} \hbar}{T}$ and disregarding the resonances, there are two distinct values of $\epsilon$ which would give the same value of $E$. Of these, the lower value would correspond to a normal physical state energy in practice whereas the higher value would be associated with a state with an enormous temporal oscillation factor. We expect that such a state would not be created in the laboratory under normal situations, because the scattering amplitudes to go to such states oscillate very rapidly in time and this would lead to cancellations to zero in detailed scattering calculations.

As far as the resonances go, these give stationary solutions which differ by at most a sign to one of the two mentioned above, and so do not represent new states and can be ignored.

An interesting question is whether every eigenfunction of the continuous time equation (34) generates a stationary state solution to (32) of the form (33). The answer is no if we require $\epsilon$ to be real. For energy eigenvalues greater than $\frac{\sqrt{3} \hbar}{T}$, solutions to (35) will develop an imaginary component, giving discrete time wavefunctions which grow or decay exponentially, and such wave-functions cannot be regarded as being stationary. An analogous cut-off phenomenon will be seen when we discuss the Klein-Gordon equation in $§ 5$.

To find a conserved charge we first rewrite the equation of motion (32) in the form

$$
\begin{gathered}
(3 i \hbar-T \vec{S}) \Psi_{n+1}=(3 i \hbar+T \vec{S}) \Psi_{n-1}+4 T \vec{S} \Psi_{n} \\
\Psi_{n+1}^{*}(-3 i \hbar-T \overleftarrow{S}) \underset{c}{=} \Psi_{n-1}^{*}(-3 i \hbar+T \overleftarrow{S})+4 T \Psi_{n}^{*} \overleftarrow{S}
\end{gathered}
$$

where $\vec{S} \equiv \frac{-\hbar^{2}}{2 m} \vec{\nabla}^{2}+V(\mathbf{x})$. Now consider the global gauge transformation

$$
\Psi_{n} \rightarrow e^{i \theta} \Psi_{n}, \quad \Psi_{n}^{*} \rightarrow e^{-i \theta} \Psi_{n}^{*}
$$

and apply the Maeda-Noether theorem discussed in $\S 3$. Then we find the invariant of the motion

$$
Q^{n} \equiv \int d^{3} \mathbf{x}\left\{\Psi_{n}^{*}\left(\frac{1}{2}+\frac{i T}{6 \hbar} \vec{S}\right) \Psi_{n+1}+\Psi_{n+1}^{*}\left(\frac{1}{2}-\overleftarrow{S} \frac{i T}{6 \hbar}\right) \Psi_{n}\right\}
$$

which reduces to the standard total probability in the limit $T \rightarrow 0$. With the equations of motion (37) we find

$$
Q^{n} \underset{c}{=} Q^{n-1}
$$

From the charge (39) we construct the charge and current densities, given by

$$
\begin{aligned}
& \rho_{n}=\Psi_{n}^{*}\left(\frac{1}{2}+\frac{i T}{6 \hbar} \vec{S}\right) \Psi_{n+1}+\Psi_{n+1}^{*}\left(\frac{1}{2}-\overleftarrow{S} \frac{i T}{6 \hbar}\right) \Psi_{n} \\
& \mathbf{j}_{n}=\frac{-i \hbar}{12 m}\left\{\Psi_{n+1}^{*} \overleftrightarrow{\nabla} \Psi_{n}+4 \Psi_{n}^{*} \overleftrightarrow{\nabla} \Psi_{n}+\Psi_{n}^{*} \overleftrightarrow{\nabla} \Psi_{n-1}\right\}
\end{aligned}
$$

which satisfy the discrete time equation of continuity

$$
\frac{\rho_{n}-\rho_{n-1}}{T}+\nabla \cdot \mathbf{j}_{n}=0
$$


The density $\rho_{n}$ above is not positive definite except in the limit $T \rightarrow 0$, when the normal density $\Psi^{*} \Psi$ is recovered. This is analogous to what happens in continuous time mechanics to the charge density for the charged Klein-Gordon equation before we take the limit $c \rightarrow \infty$. One of the reasons occasionally cited for Schrödinger's rejection of a relativistic wave equation in 1925-26 is because of just this point, there being no evidence at that time for positive electron charge densities. We imagine that if discrete time mechanics had been the accepted classical mechanical paradigm prior to wave mechanics then the Born probability interpretation of Schrödinger wave mechanics would probably not have been proposed.

If we wish to couple electromagnetic fields in a gauge invariant way to the Schrödinger equation we need to choose a modified virtual path. Such a path is used for the charged Klein-Gordon equation discussed below, and so this is left as an exercise in the case of the Schrödinger equation.

\section{The Klein-Gordon field}

\subsection{The continuous time system}

An important system is the free scalar field system with Lagrange density

$$
\mathcal{L} \equiv \frac{1}{2} \partial_{\mu} \varphi \partial^{\mu} \varphi-\frac{1}{2} \mu^{2} \varphi^{2}
$$

where we shall take $c=\hbar=1$ and three spatial dimensions. Hamilton's principal function is given by

$$
\begin{aligned}
S^{n}= & \frac{1}{2} \int \frac{d^{3} \mathbf{p}}{(2 \pi)^{3}} \frac{p_{0}}{\sin \left(p_{0} T\right)} \int d^{3} \mathbf{x} d^{3} \mathbf{y} \mathbf{e}^{i \mathbf{p} \cdot(\mathbf{x}-\mathbf{y})} \times \\
& \left\{\left[\varphi_{n+1}(\mathbf{x}) \varphi_{n+1}(\mathbf{y})+\varphi_{n}(\mathbf{x}) \varphi_{n}(\mathbf{y})\right] \cos \left(p_{0} T\right)-2 \varphi_{n+1}(\mathbf{x}) \varphi_{n}(\mathbf{y})\right\},
\end{aligned}
$$

where $p_{0} \equiv \sqrt{\mathbf{p} \cdot \mathbf{p}+\mu^{2}}$. Now compare this principal function with that for the continuous time mechanics harmonic oscillator, where the phenomenon of recurrence occurs, as discussed in Paper I. We note that in the field theory, recurrence occurs in momentum space. By inspection of (44), singularities in the momentum space integrand appear to arise whenever $p_{0} T=m \pi, m=1,2, \ldots$ To understand this here we first construct the conjugate momenta in terms of the end-point field values using (13). From the principal function (44) we find

$$
\pi_{n}(\mathbf{x})=\int \frac{d^{3} \mathbf{p}}{(2 \pi)^{3}} \frac{p_{0}}{\sin \left(p_{0} T\right)} \int d^{3} \mathbf{y} \mathbf{e}^{i \mathbf{p} \cdot(\mathbf{x}-\mathbf{y})}\left\{\varphi_{n+1}(\mathbf{y})-\varphi_{n}(\mathbf{y}) \cos \left(p_{0} T\right)\right\}
$$

which satisfies the expected relation

$$
\lim _{T \rightarrow 0} \pi_{n}(\mathbf{x})=\dot{\varphi}_{n}(\mathbf{x})
$$

if we assume the fields vary smoothly with time. 
Important constructions are the momentum fields

$$
\begin{aligned}
A(\mathbf{p}) & \equiv \int d^{3} \mathbf{x} e^{i p x}\left\{p_{0} \varphi+i \pi\right\} \\
A^{*}(\mathbf{p}) & \equiv \int d^{3} \mathbf{x} e^{-i p x}\left\{p_{0} \varphi-i \pi\right\},
\end{aligned}
$$

which are time independent modulo the Klein-Gordon equation. Using (45) we find

$$
A_{n}(\mathbf{p}) \equiv \frac{i p_{0}}{\sin \left(p_{0} T\right)} \int d^{3} \mathbf{x} e^{i n p_{0} T-i \mathbf{p} \cdot \mathbf{x}}\left\{\varphi_{n+1}(\mathbf{x})-e^{i p_{0} T} \varphi_{n}(\mathbf{x})\right\}
$$

and similarly for its complex conjugate. The time independence of the above momentum fields means that

$$
A_{n}(\mathbf{p}) \underset{c}{=} A_{n+1}(\mathbf{p}) .
$$

Moreover, the momentum fields are not singular, as is evident from (47, 48), so that in the limit $p_{0} T \rightarrow m \pi$ the apparent singularity in $A_{n}(\mathbf{p})$ must be cancelled by recurrence behaviour in the Fourier transforms

$$
\tilde{\varphi}_{n}(\mathbf{p}) \equiv \int d^{3} \mathbf{x} e^{-i \mathbf{p} \cdot \mathbf{x}} \varphi_{n}(\mathbf{x}) .
$$

We deduce from (50) that the Fourier transformed fields $\tilde{\varphi}_{n}(\mathbf{p})$ satisfy the equation of motion

$$
\tilde{\varphi}_{n+1}(\mathbf{p})+\tilde{\varphi}_{n-1}(\mathbf{p}) \underset{c}{=} 2 \cos \left(p_{0} T\right) \tilde{\varphi}_{n}(\mathbf{p}) .
$$

This shows that the continuous time Klein-Gordon field behaves like the discrete time harmonic oscillator discussed in Paper $I$ and the discrete time Klein-Gordon field discussed next, but with one important difference. The magnitude of the factor $\cos \left(p_{0} T\right)$ in the above equation of motion never exceeds unity and this ensures that the motion is never hyperbolic. There is no need therefore for a momentum cut-off in the continuous time theory.

\subsection{The discrete time Klein-Gordon field}

We now consider discretising the continuous time system with Lagrange density (43) . The virtual paths are of the form

$$
\tilde{\varphi}(\mathbf{x}) \equiv \lambda \varphi_{n+1}(\mathbf{x})+\bar{\lambda} \varphi_{n}(\mathbf{x}), \quad 0 \leq \lambda \leq 1,
$$

where $\bar{\lambda}=1-\lambda$. With this choice of virtual path the time derivative $\partial_{t}$ may be replaced by the operator $T^{-1} \partial_{\lambda}$, which acts on $\tilde{\varphi}$ as a difference operator, viz

$$
\partial_{t} \tilde{\varphi}=\frac{\varphi_{n+1}-\varphi_{n}}{T} .
$$

The system function $F^{n}=F\left(\varphi_{n}, \varphi_{n+1}, \nabla \varphi_{n}, \nabla \varphi_{n+1}\right)$ is then the spatial integral

$$
F^{n}=\int d^{3} \mathbf{x} \mathcal{F}^{n}(\mathbf{x})
$$


of the system function density $\mathcal{F}^{n}(\mathbf{x})$, given by

$$
\mathcal{F}^{n}(\mathbf{x}) \equiv T \int_{0}^{1} d \lambda \mathcal{L}\left(\tilde{\varphi}, \partial_{\mu} \tilde{\varphi}\right)
$$

We find

$$
\begin{aligned}
\mathcal{F}^{n}= & \frac{\left(\varphi_{n+1}-\varphi_{n}\right)^{2}}{2 T}-\frac{T}{6}\left(\left|\nabla \varphi_{n+1}\right|^{2}+\nabla \varphi_{n+1} \cdot \nabla \varphi_{n}+\left|\nabla \varphi_{n}\right|^{2}\right) \\
& -\frac{\mu^{2} T}{6}\left(\varphi_{n+1}{ }^{2}+\varphi_{n+1} \varphi_{n}+\varphi_{n}{ }^{2}\right)
\end{aligned}
$$

from which Cadzow's equation of motion (20) gives

$$
\frac{\varphi_{n+1}-2 \varphi_{n}+\varphi_{n-1}}{T^{2}}+\vec{K} \frac{\left(\varphi_{n+1}+4 \varphi_{n}+\varphi_{n-1}\right)}{6}=0
$$

where $\vec{K} \equiv-\nabla^{2}+\mu^{2}$. This equation can be written in the form

$$
\vec{D} \varphi_{n+1}+4 \vec{D} \varphi_{n}+\vec{D} \varphi_{n-1}=\frac{6}{c} \varphi_{n}
$$

where

$$
\vec{D} \equiv \frac{6+T^{2} \vec{K}}{6 T}
$$

which is useful for proving various quantities are conserved. For example, the linear momentum obtained using (24) is found to be

$$
\mathbf{P}^{n}=-\int d^{3} \mathbf{x} \varphi_{n} \overleftarrow{\nabla} \vec{D} \varphi_{n+1}
$$

and using (59) we find

$$
\mathbf{P}^{n}=\mathbf{P}^{n-1}
$$

Likewise, the orbital angular momentum is conserved and given by

$$
\mathbf{L}^{n}=-\int d^{3} \mathbf{x}\left(\mathbf{x} \times \nabla \varphi_{n}\right) \vec{D} \varphi_{n+1} .
$$

These invariants of the motion exist because we have not destroyed Euclidean invariance, that is, there is still spatial translational and rotational invariance in our approach. This will occur in all our discrete time field models based on special relativistic Lagrangians. We note that a similar equation was found for the scalar field in the $\kappa$-Poincaré theory of Lukierski et al. [8], with the important differences that their lattice parameter corresponding to our $T$ is imaginary and that they are dealing with continuous time throughout.

We turn now to particle-like solutions to the equation of motion. Consider the Fourier transform

$$
\tilde{\varphi}_{n}(\mathbf{p})=\int d^{3} \mathbf{x} e^{-i \mathbf{p} \cdot \mathbf{x}} \varphi_{n}(\mathbf{x})
$$


The equation of motion (59) becomes

$$
\beta \tilde{\varphi}_{n+1}+4 \beta \tilde{\varphi}_{n}+\beta \tilde{\varphi}_{n-1}=\frac{6}{T} \tilde{\varphi}_{n}
$$

where $\beta \equiv \frac{6+T^{2} E^{2}}{6 T}$ and $E \equiv \sqrt{\mu^{2}+\mathbf{p} \cdot \mathbf{p}}$. Now define the momentum functions

$$
\begin{aligned}
& a_{n}(\mathbf{p})=i \beta e^{i n \theta}\left[\tilde{\varphi}_{n+1}-e^{i \theta} \tilde{\varphi}_{n}\right], \\
& a_{n}^{*}(\mathbf{p})=-i \beta e^{-i n \theta}\left[\tilde{\varphi}_{n+1}^{*}-e^{-i \theta} \tilde{\varphi}_{n}^{*}\right],
\end{aligned}
$$

where $\cos \theta=\eta, \sin \theta=\sqrt{1-\eta^{2}}$ with $\eta$ given by the ratio

$$
\eta=\frac{\alpha}{\beta}, \quad \alpha=\frac{6-2 T^{2} E^{2}}{6 T} .
$$

These are closely related to the continuous time momentum functions (49) and to the ladder operators discussed in Paper $I$ when we identify $e^{i \theta}=\mu \equiv \eta+i \sqrt{1-\eta^{2}}$. Particle states in discrete time field theory correspond to elliptic type wave behaviour, which requires $|\eta|<1$. Otherwise, physically unacceptable hyperbolic behaviour occurs, as discussed in Paper $I$. This is equivalent to the condition

$$
T E<2 \sqrt{3},
$$

i.e.

$$
c \sqrt{\mathbf{p} \cdot \mathbf{p}+\mu^{2} c^{2}}<\frac{2 \sqrt{3} \hbar}{T}
$$

in Standard International units. The fundamental conclusion from this analysis is that energy and momentum are bounded above for physical particle states in our approach. In other words, in discrete time field theory, the spectrum of acceptable particle states has a natural cut-off which can be made as large enough to avoid a violation of observed particle data as necessary by choosing the time interval $T$ small enough.

An interesting point is that for a given $T$, there can be no scalar particle species with rest mass greater than $2 \sqrt{3} \hbar / T c^{2}$, but this cannot be regarded as of interest to experimentalists at this time.

We note that by construction the momentum fields are time independent and satisfy

$$
a_{n}(\mathbf{p}) \underset{c}{=} a_{n-1}(\mathbf{p}), \quad a_{n}^{*}(\mathbf{p}) \underset{c}{=} a_{n-1}^{*}(\mathbf{p}) .
$$

This allows us to construct a Logan invariant [7] of the motion

$$
C^{n} \equiv \int \frac{d^{3} \mathbf{p}}{(2 \pi)^{3}} \theta\left(\frac{12}{T^{2}}-\mu^{2}-\mathbf{p} \cdot \mathbf{p}\right) a_{n}^{*}(\mathbf{p}) a_{n}(\mathbf{p})
$$

which in the limit $T \rightarrow 0$ becomes the standard field theory Hamiltonian, using the result

$$
\lim _{T \rightarrow 0, n T \rightarrow t} a_{n}(\mathbf{p})=i \int d^{3} \mathbf{x} e^{i E t-i \mathbf{p} \cdot \mathbf{x}}[\dot{\varphi}(t, \mathbf{x})-i E \varphi(t, \mathbf{x})]=A(\mathbf{p}) .
$$

for those trajectories for which the limit exists. It is important to understand however that there is no notion of a Hamiltonian in our approach, since there is no possibility of infinitesimal translation in time, except in the above limit. 


\section{The free charged Klein Gordon equation}

For this system we consider the continuous time Lagrange density

$$
\mathcal{L}=\partial_{\mu} \varphi^{*} \partial^{\mu} \varphi-\mu^{2} \varphi^{*} \varphi
$$

The virtual paths are given as before by

$$
\tilde{\varphi} \equiv \lambda \varphi_{n+1}+\bar{\lambda} \varphi_{n}, \quad \tilde{\varphi}^{*} \equiv \lambda \varphi_{n+1}^{*}+\bar{\lambda} \varphi_{n}^{*}, \quad 0 \leq \lambda \leq 1
$$

where we suppress the dependence on $\mathbf{x}$. Then the system function density is found to be

$$
\begin{aligned}
\mathcal{F}^{n}= & \frac{\left|\varphi_{n+1}-\varphi_{n}\right|^{2}}{T}-\frac{T}{6}\left\{2\left|\nabla \varphi_{n+1}\right|^{2}+2\left|\nabla \varphi_{n}\right|^{2}+\nabla \varphi_{n+1}^{*} \cdot \nabla \varphi_{n}+\nabla \varphi_{n}^{*} \cdot \nabla \varphi_{n+1}\right\} \\
& -\frac{\mu^{2} T}{6}\left\{2\left|\varphi_{n+1}\right|^{2}+2\left|\varphi_{n}\right|^{2}+\varphi_{n+1}^{*} \varphi_{n}+\varphi_{n}^{*} \varphi_{n+1}\right\} .
\end{aligned}
$$

From this Cadzow's equations of motion give

$$
\begin{aligned}
& \frac{\varphi_{n+1}-2 \varphi_{n}+\varphi_{n-1}}{T^{2}}+\vec{K} \frac{\left(\varphi_{n+1}+4 \varphi_{n}+\varphi_{n-1}\right)}{6}=0 \\
& \frac{\varphi_{n+1}^{*}-2 \varphi_{n}^{*}+\varphi_{n-1}^{*}}{T^{2}}+\vec{K} \frac{\left(\varphi_{n+1}^{*}+4 \varphi_{n}^{*}+\varphi_{n-1}^{*}\right)}{6}=0,
\end{aligned}
$$

which can be written in the form

$$
\begin{aligned}
& \vec{D} \varphi_{n+1}+4 \vec{D} \varphi_{n}+\vec{D} \varphi_{n-1}=\frac{6}{T} \varphi_{n} \\
& \vec{D} \varphi_{n+1}^{*}+4 \vec{D} \varphi_{n}^{*}+\vec{D} \varphi_{n-1}^{*}=\frac{6}{T} \varphi_{n}^{*}
\end{aligned}
$$

where $\vec{D}$ is given by $(60)$.

The linear and angular momenta are easy to construct so we turn to the new feature, global gauge invariance. The system function density (76) is invariant to a global gauge transformation of the fields, i.e.

$$
\varphi_{n} \rightarrow \varphi_{n}^{\prime}=e^{-i \chi} \varphi_{n}, \quad \varphi_{n}^{*} \rightarrow \varphi_{n}^{* \prime}=e^{i \chi} \varphi_{n}^{*}
$$

where $\chi$ is independent of time and space, so using the Maeda-Noether theorem discussed in $\S 3$ we find the conserved charge

$$
\begin{aligned}
Q^{n} & \equiv i \int d^{3} \mathbf{x}\left\{\varphi_{n}^{*} \vec{D} \varphi_{n+1}-\varphi_{n+1}^{*} \overleftarrow{D} \varphi_{n}\right\} \\
& =i \int d^{3} \mathbf{x}\left\{\varphi_{n}^{*} \overleftarrow{D} \varphi_{n+1}-\varphi_{n+1}^{*} \vec{D} \varphi_{n}\right\}
\end{aligned}
$$

This is real and global gauge invariant. Using the equations of motion we find

$$
Q^{n} \underset{c}{=} Q^{n-1}
$$


By inspection there are two possible candidates for a charge density, denoted by $\rho^{(-)}$and $\rho^{(+)}$, given by

$$
\begin{aligned}
\rho_{n}^{(-)} & \equiv i \varphi_{n}^{*} \vec{D} \varphi_{n+1}-i \varphi_{n+1}^{*} \overleftarrow{D} \varphi_{n} \\
\rho_{n}^{(+)} & \equiv i \varphi_{n}^{*} \overleftarrow{D} \varphi_{n+1}-i \varphi_{n+1}^{*} \vec{D} \varphi_{n}
\end{aligned}
$$

These are related by a total divergence, that is

$$
\rho_{n}^{(+)}=\rho^{(-)}{ }_{n}+\frac{i T}{6} \nabla \cdot\left[\varphi_{n}^{*} \overleftrightarrow{\nabla} \varphi_{n+1}+\varphi_{n+1}^{*} \overleftrightarrow{\nabla} \varphi_{n}\right]
$$

so that they give the same total charge $Q^{n}$. Using the equations of motion we find

$$
\begin{aligned}
& \frac{\rho_{n}^{(-)}-\rho_{n-1}^{(-)}}{T}=\frac{i}{c} \nabla \cdot\left[\varphi_{n-1}^{*} \overleftrightarrow{\nabla} \varphi_{n}+\varphi_{n}^{*} \overleftrightarrow{\nabla} \varphi_{n-1}+4 \varphi_{n}^{*} \overleftrightarrow{\nabla} \varphi_{n}\right] \\
& \frac{\rho_{n}^{(+)}-\rho_{n-1}^{(+)}}{T}=\frac{i}{6} \nabla \cdot\left[\varphi_{n}^{*} \overleftrightarrow{\nabla} \varphi_{n+1}+\varphi_{n+1}^{*} \overleftrightarrow{\nabla} \varphi_{n}+4 \varphi_{n}^{*} \overleftrightarrow{\nabla} \varphi_{n}\right],
\end{aligned}
$$

which are discrete time versions of the charge continuity equation, with corresponding charge currents given by

$$
\begin{aligned}
\mathbf{j}_{n}^{(-)} & =\frac{-i}{6}\left[\varphi_{n-1}^{*} \overleftrightarrow{\nabla} \varphi_{n}+\varphi_{n}^{*} \overleftrightarrow{\nabla} \varphi_{n-1}+4 \varphi_{n}^{*} \overleftrightarrow{\nabla} \varphi_{n}\right] \\
\mathbf{j}_{n}^{(+)} & =\frac{-i}{6}\left[\varphi_{n}^{*} \overleftrightarrow{\nabla} \varphi_{n+1}+\varphi_{n+1}^{*} \overleftrightarrow{\nabla} \varphi_{n}+4 \varphi_{n}^{*} \overleftrightarrow{\nabla} \varphi_{n}\right]
\end{aligned}
$$

\section{Maxwell's equations}

\subsection{Charge free equations}

We retain our unit system such that $c=\hbar=1$. Our discrete time formulation of charge free Maxwell's equations starts with the electromagnetic potentials $\phi$ and $\mathbf{A}$ which are used to construct the physical electric and magnetic fields $\mathbf{E}$ and $\mathbf{B}$. A clear distinction has to be made here between the nature of the electric scalar potential $\phi$ and the magnetic vector potential $\mathbf{A}$. The former is associated with the temporal interval or link connecting times $t_{n}$ and $t_{n+1}$, whereas the latter is associated with the times themselves. This distinction also manifests itself in the difference between the physical electric field $\mathbf{E}$ and the magnetic field $\mathbf{B}$, which are likewise associated with temporal links and endpoints respectively.

Our definitions are as follows:

The electric scalar potential associated with the link connecting time $t_{n}$ and $t_{n+1}$ at spatial position $\mathbf{x}$ will be denoted by the symbol $\phi_{n}(\mathbf{x})$, rather than by (say) $\phi_{n+\frac{1}{2}}(\mathbf{x})$. Although our notation suggests a bias towards $t_{n}$ at the expense of $t_{n+1}$, this is not really the case. The virtual path $\tilde{\phi}_{n}$ for the electric potential is defined by

$$
\tilde{\phi}_{n}(\mathbf{x}) \equiv \phi_{n}(\mathbf{x}), \quad 0 \leq \lambda \leq 1 .
$$


On the other hand the magnetic vector potential associated at time $t_{n}$ at spatial position $\mathbf{x}$ will be denoted by $\mathbf{A}_{n}(\mathbf{x})$ and its associated virtual path $\tilde{\mathbf{A}}_{n}$ is defined by

$$
\tilde{\mathbf{A}}_{n}(\mathbf{x}) \equiv \lambda \mathbf{A}_{n+1}(\mathbf{x})+\bar{\lambda} \mathbf{A}_{n}(\mathbf{x}), \quad 0 \leq \lambda \leq 1
$$

A local gauge transformation involves the gauge functions $\chi$, which are associated with endpoints rather than links. A gauge function value at time $t_{n}$ and position $\mathbf{x}$ will be denoted by $\chi_{n}(\mathbf{x})$ and is assumed differentiable with respect to $\mathbf{x}$. We shall see in the next section on charged scalar field electrodynamics that the virtual path for the gauge fields is given by

$$
\tilde{\chi}_{n}(\mathbf{x}) \equiv \lambda \chi_{n+1}(\mathbf{x})+\bar{\lambda} \chi_{n}(\mathbf{x}), \quad 0 \leq \lambda \leq 1
$$

A local gauge transformation is defined here by the replacements

$$
\tilde{\mathbf{A}}_{n}^{\mu} \rightarrow \tilde{\mathbf{A}}_{n}^{\prime \mu}=\tilde{\mathbf{A}}_{n}^{\mu}+\partial^{\mu} \tilde{\chi}_{n}
$$

which reduce to the transformations

$$
\begin{aligned}
& \phi_{n} \rightarrow \phi_{n}^{\prime}=\phi_{n}+\frac{\chi_{n+1}-\chi_{n}}{T} \\
& \mathbf{A}_{n} \rightarrow \mathbf{A}_{n}^{\prime}=\mathbf{A}_{n}-\nabla \chi_{n} .
\end{aligned}
$$

Turning now to the physical fields, we define the gauge invariant electric and magnetic fields via the potentials:

$$
\mathbf{E}_{n} \equiv-\nabla \phi_{n}-\frac{\left(\mathbf{A}_{n+1}-\mathbf{A}_{n}\right)}{T}, \quad \mathbf{B}_{n} \equiv \nabla \times \mathbf{A}_{n}
$$

which give the homogeneous Maxwell equations

$$
\nabla \cdot \mathbf{B}_{n}=0, \quad \nabla \times \mathbf{E}_{n}+\frac{\left(\mathbf{B}_{n+1}-\mathbf{B}_{n}\right)}{T}=\mathbf{0}
$$

The gauge invariant system function density for the charge free system is defined as the integral

$$
\mathcal{F}^{n} \equiv T \int_{0}^{1} d \lambda\left(-\frac{1}{4} \tilde{F}_{n \mu \nu} \tilde{F}_{n}^{\mu \nu}\right),
$$

where $\tilde{F}_{n}^{\mu \nu} \equiv \partial^{\mu} \tilde{A}_{n}^{\nu}-\partial^{\nu} \tilde{A}_{n}^{\mu}$ are the components of the Faraday tensor. This gives

$$
\mathcal{F}^{n}=\frac{T}{2} \mathbf{E}_{n}^{2}-\frac{T}{6}\left(\mathbf{B}_{n+1}^{2}+\mathbf{B}_{n+1} \cdot \mathbf{B}_{n}+\mathbf{B}_{n}^{2}\right) .
$$

Applying Cadzow's equation to (101) we find

$$
\nabla \cdot \mathbf{E}_{n} \underset{c}{=} 0, \quad \frac{\left(\mathbf{E}_{n}-\mathbf{E}_{n-1}\right)}{T}=\nabla \times \frac{\left(\mathbf{B}_{n+1}+4 \mathbf{B}_{n}+\mathbf{B}_{n-1}\right)}{6} .
$$


Using (99) and (102) we find the physical electromagnetic fields satisfy the discrete time massless Klein-Gordon equations

$$
\begin{aligned}
& \frac{\mathbf{E}_{n+1}-2 \mathbf{E}_{n}+\mathbf{E}_{n-1}}{T^{2}}-\nabla^{2} \frac{\left(\mathbf{E}_{n+1}+4 \mathbf{E}_{n}+\mathbf{E}_{n-1}\right)}{6}=\mathbf{0}, \\
& \frac{\mathbf{B}_{n+1}-2 \mathbf{B}_{n}+\mathbf{B}_{n-1}}{T^{2}}-\nabla^{2} \frac{\left(\mathbf{B}_{n+1}+4 \mathbf{B}_{n}+\mathbf{B}_{n-1}\right)}{6}=\mathbf{0} .
\end{aligned}
$$

Turning to the gauge potentials, we define the discrete time Lorentz gauge by the condition

$$
\frac{\phi_{n}-\phi_{n-1}}{T}+\nabla \cdot \frac{\left(\mathbf{A}_{n+1}+4 \mathbf{A}_{n}+\mathbf{A}_{n-1}\right)}{6}=0
$$

and then the gauge potentials also satisfy the discrete time massless Klein-Gordon equations

$$
\begin{gathered}
\frac{\mathbf{A}_{n+1}-2 \mathbf{A}_{n}+\mathbf{A}_{n-1}}{T^{2}}-\nabla^{2} \frac{\left(\mathbf{A}_{n+1}+4 \mathbf{A}_{n}+\mathbf{A}_{n-1}\right)}{6}=\mathbf{0} \\
\frac{\phi_{n+1}-2 \phi_{n}+\phi_{n-1}}{T^{2}}-\nabla^{2} \frac{\left(\phi_{n+1}+4 \phi_{n}+\phi_{n-1}\right)}{6}=0 .
\end{gathered}
$$

The total linear momentum for the free electromagnetic fields is found to be

$$
\mathbf{P}^{n}=\int d^{3} \mathbf{x}\left\{\mathbf{E}_{n} \times \mathbf{B}_{n}+\frac{T}{6} B_{n}^{i} \vec{\nabla} B_{n+1}^{i}\right\}
$$

Then using the equations of motion we find

$$
\mathbf{P}^{n}=\mathbf{P}^{n-1}
$$

as expected. In the limit $T \rightarrow 0$ the above expression reduces to the Poynting vector.

\subsection{Maxwell's equations in the presence of charges}

In the presence of electric charges the system function density (101) is replaced by

$$
\mathcal{F}^{n}=\frac{T}{2} \mathbf{E}_{n}^{2}-\frac{T}{6}\left(\mathbf{B}_{n+1}^{2}+\mathbf{B}_{n+1} \cdot \mathbf{B}_{n}+\mathbf{B}_{n}^{2}\right)-T \phi_{n} \rho_{n}+T \mathbf{A}_{n} \cdot \mathbf{j}_{n}
$$

where $\rho_{n}(\mathbf{x})$ and $\mathbf{j}_{n}(\mathbf{x})$ are the discrete time charge density and charge current respectively. The homogeneous equations (99) remain unaltered but now the equations of motion become

$$
\nabla \cdot \mathbf{E}_{n}=\rho_{n}, \quad \nabla \times \frac{\left(\mathbf{B}_{n+1}+4 \mathbf{B}_{n}+\mathbf{B}_{n-1}\right)}{6}-\frac{\left(\mathbf{E}_{n}-\mathbf{E}_{n-1}\right)}{T}=\mathbf{j}_{n} .
$$

These equations are consistent with the equation of continuity for electric charge given by

$$
\frac{\rho_{n}-\rho_{n-1}}{T}+\nabla \cdot \mathbf{j}_{n}=0
$$


Finally, in a discrete time Lorentz gauge (105) the potentials satisfy the equations

$$
\begin{aligned}
& \frac{\mathbf{A}_{n+1}-2 \mathbf{A}_{n}+\mathbf{A}_{n-1}}{T^{2}}-\nabla^{2} \frac{\left(\mathbf{A}_{n+1}+4 \mathbf{A}_{n}+\mathbf{A}_{n-1}\right)}{6}=\mathbf{j}_{n}, \\
& \frac{\phi_{n+1}-2 \phi_{n}+\phi_{n-1}}{T^{2}}-\nabla^{2} \frac{\left(\phi_{n+1}+4 \phi_{n}+\phi_{n-1}\right)}{6}=\frac{\left(\rho_{n+1}+4 \rho_{n}+\rho_{n-1}\right)}{6} .
\end{aligned}
$$

\section{Scalar field electrodynamics}

We are now in a position to discuss the coupling of the Maxwell potentials to the charged Klein-Gordon field. In this case the virtual paths for the charged scalar field and its complex conjugate have to be modified so as to ensure that the system function is gauge invariant. We define

$$
\begin{aligned}
\tilde{\varphi} & \equiv \lambda e^{i q \phi_{n} T \bar{\lambda}} \varphi_{n+1}+\bar{\lambda} e^{-i q \phi_{n} T \lambda} \varphi_{n}, \\
\tilde{\varphi}^{*} & \equiv \lambda e^{-i q \phi_{n} T \bar{\lambda}} \varphi_{n+1}^{*}+\bar{\lambda} e^{i q \phi_{n} T \lambda} \varphi_{n}^{*}, \quad \bar{\lambda}=1-\lambda: \quad 0 \leq \lambda \leq 1 .
\end{aligned}
$$

where $\phi_{n}$ is the scalar potential discussed in the previous section. Then the gauge transformations are given by $(96,97)$ and by

$$
\varphi_{n}^{\prime}=e^{-i q \chi_{n}} \varphi_{n}, \quad \varphi_{n+1}^{\prime}=e^{-i q \chi_{n+1}} \varphi_{n+1},
$$

which leads to the compact form

$$
\tilde{\varphi}^{\prime}=e^{-i q \tilde{\chi}} \tilde{\varphi}, \quad \tilde{\varphi}^{* \prime}=e^{i q \tilde{\chi}} \tilde{\varphi}^{*} .
$$

Now define the gauge covariant derivatives

$$
\tilde{D}_{\mu} \tilde{\varphi} \equiv\left(\partial_{\mu}+i q \tilde{A}_{\mu}\right) \tilde{\varphi}
$$

and its complex conjugate. Under a gauge transformation we find

$$
\left(\tilde{D}_{\mu} \tilde{\varphi}\right)^{\prime} \equiv e^{-i q \tilde{\chi}} \tilde{D}_{\mu} \tilde{\varphi}
$$

Next we construct the gauge invariant Lagrange density

$$
\begin{aligned}
\mathcal{L}^{n} & =\left(\tilde{D}_{\mu} \tilde{\varphi}\right)^{*} \tilde{D}^{\mu} \tilde{\varphi}-\mu^{2} \tilde{\varphi}^{*} \tilde{\varphi} \\
& =\left(\tilde{D}_{0} \tilde{\varphi}\right)^{*} \tilde{D}^{0} \tilde{\varphi}-(\tilde{\mathbf{D}} \tilde{\varphi})^{*} \cdot \tilde{\mathbf{D}} \tilde{\varphi}-\mu^{2} \tilde{\varphi}^{*} \tilde{\varphi}
\end{aligned}
$$

where

$$
\tilde{D}_{0} \tilde{\varphi} \equiv\left(\frac{1}{T} \partial_{\lambda}+i q \phi_{n}\right) \tilde{\varphi}=\frac{e^{i q \phi_{n} T \bar{\lambda}} \varphi_{n+1}-e^{-i q \phi_{n} T \lambda} \varphi_{n}}{T}
$$

and

$$
\begin{aligned}
\tilde{\mathbf{D}} \tilde{\varphi} \equiv & (\nabla-i q \tilde{\mathbf{A}}) \tilde{\varphi} \\
= & i q T \lambda \bar{\lambda} \nabla \phi_{n}\left\{e^{i q \phi_{n} T \bar{\lambda}} \varphi_{n+1}-e^{-i q \phi_{n} T \lambda} \varphi_{n}\right\} \\
& +\lambda e^{i q \phi_{n} T \bar{\lambda}} \nabla \varphi_{n+1}+\bar{\lambda} e^{-i q \phi_{n} T \lambda} \nabla \varphi_{n}-i q \tilde{\mathbf{A}} \tilde{\varphi}
\end{aligned}
$$


The system function is constructed as before from the integral

$$
\mathcal{F}^{n}=T \int_{0}^{1} d \lambda\left\{\left(\tilde{D}_{0} \tilde{\varphi}\right)^{*} \tilde{D}_{0} \tilde{\varphi}-(\tilde{\mathbf{D}} \tilde{\varphi})^{*} \cdot \tilde{\mathbf{D}} \tilde{\varphi}-\mu^{2} \tilde{\varphi}^{*} \tilde{\varphi}\right\}
$$

which gives

$$
\begin{aligned}
\mathcal{F}^{n}= & \left(\frac{1}{T}-\frac{q^{2} T^{3}}{30} \nabla \phi_{n} \cdot \nabla \phi_{n}\right)\left|U_{n} \varphi_{n+1}-\varphi_{n}\right|^{2} \\
& -\frac{T}{6}\left\{2\left|\nabla \varphi_{n+1}\right|^{2}+2\left|\nabla \varphi_{n}\right|^{2}+U_{n}^{*} \nabla \varphi_{n+1}^{*} \cdot \nabla \varphi_{n}+\nabla \varphi_{n}^{*} \cdot \nabla \varphi_{n+1} U_{n}\right\} \\
& -\frac{\mu^{2} T}{6}\left\{2\left|\varphi_{n+1}\right|^{2}+2\left|\varphi_{n}\right|^{2}+U_{n}^{*} \varphi_{n+1}^{*} \varphi_{n}+\varphi_{n}^{*} \varphi_{n+1} U_{n}\right\} \\
& +\frac{i q T^{2}}{12}\left\{\left(U_{n}^{*} \varphi_{n+1}^{*}-\varphi_{n}^{*}\right) \nabla \phi_{n} \cdot\left(U_{n} \nabla \varphi_{n+1}+\nabla \varphi_{n}\right)\right\} \\
& \left.+\nabla \phi_{n} \cdot\left(U_{n}^{*} \nabla \varphi_{n+1}^{*}+\nabla \varphi_{n}^{*}\right)\left(U_{n} \varphi_{n+1}-\varphi_{n}\right)\right\} \\
& \left.+\left[\left(3 \mathbf{A}_{n+1}+2 \mathbf{A}_{n}\right) U_{n}^{*} \varphi_{n+1}^{*}+\left(2 \mathbf{A}_{n+1}+3 \mathbf{A}_{n}\right) \varphi_{n}^{*}\right] \cdot \nabla \varphi_{n}\left(U_{n} \varphi_{n+1}-\varphi_{n}\right)\right\} \\
& -\frac{i q T}{12}\left\{\left(3 \mathbf{A}_{n+1}+\mathbf{A}_{n}\right) \cdot\left(\varphi_{n+1}^{*} \nabla \varphi_{n+1}^{*}-\nabla \varphi_{n+1}^{*} \varphi_{n+1}\right)\right. \\
& +\left(3 \mathbf{A}_{n}+\mathbf{A}_{n+1}\right) \cdot\left(\varphi_{n}^{*} \nabla \varphi_{n}-\nabla \varphi_{n}^{*} \varphi_{n}\right) \\
& \left.+\left(\mathbf{A}_{n+1}+\mathbf{A}_{n}\right) \cdot\left[\varphi_{n}^{*} \nabla \varphi_{n+1} U_{n}-\nabla \varphi_{n}^{*} \varphi_{n+1} U_{n}+U_{n}^{*} \varphi_{n+1}^{*} \nabla \varphi_{n}-U_{n}^{*} \nabla \varphi_{n+1}^{*} \varphi_{n}\right]\right\} \\
& -\frac{q^{2} T}{60}\left\{\left(12 \mathbf{A}_{n+1}^{2}+2 \mathbf{A}_{n}^{2}+6 \mathbf{A}_{n} \cdot \mathbf{A}_{n+1}\right)\left|\varphi_{n+1}\right|_{n}^{2}\right. \\
+ & \left(12 \mathbf{A}_{n}^{2}+2 \mathbf{A}_{n+1}^{2}+6 \mathbf{A}_{n} \cdot \mathbf{A}_{n+1}\right)\left|\varphi_{n}\right|^{2} \\
& \left.+\left(3 \mathbf{A}_{n+1}^{2}+3 \mathbf{A}_{n}^{2}+4 \mathbf{A}_{n} \cdot \mathbf{A}_{n+1}\right)\left[U_{n}^{*} \varphi_{n+1}^{*} \varphi_{n}+\varphi_{n}^{*} \varphi_{n+1} U_{n}\right]\right\}
\end{aligned}
$$

where $U_{n} \equiv e^{i q \phi_{n} T}$. This system function is gauge invariant.

The application of the resulting Cadzow's equations to specific problems is left for possible future investigation. We make the following comments in passing. First, this system function looks complicated. However, that is a matter of notation, as the expression (122) is equivalent and much more compact. There is undoubtedly much dynamical content in this system function and calculations would no doubt require approximations to be made. However we recall here Gell-Mann's dictum that it is better to make approximations to exact equations than to solve exactly approximate equations. Also, most applications would themselves be better considered from a second quantised approach, which we shall study in Paper IV of this series.

We may simplify the system function considerably by considering the special case when the external magnetic potential $\mathbf{A}_{n}$ vanishes. Then the system function reduces to

$$
\begin{aligned}
\mathcal{F}^{n}= & \left(\frac{1}{T}-\frac{q^{2} T^{3}}{30} \nabla \phi_{n} \cdot \nabla \phi_{n}\right)\left|U_{n} \varphi_{n+1}-\varphi_{n}\right|^{2} \\
& -\frac{T}{6}\left\{2\left|\nabla \varphi_{n+1}\right|^{2}+2\left|\nabla \varphi_{n}\right|^{2}+U_{n}^{*} \nabla \varphi_{n+1}^{*} \cdot \nabla \varphi_{n}+\nabla \varphi_{n}^{*} \cdot \nabla \varphi_{n+1} U_{n}\right\}
\end{aligned}
$$




$$
\begin{aligned}
& -\frac{\mu^{2} T}{6}\left\{2\left|\varphi_{n+1}\right|^{2}+2\left|\varphi_{n}\right|^{2}+U_{n}^{*} \varphi_{n+1}^{*} \varphi_{n}+\varphi_{n}^{*} \varphi_{n+1} U_{n}\right\} \\
& +\frac{i q T^{2}}{12}\left\{\begin{array}{c}
\left(U_{n}^{*} \varphi_{n+1}^{*}-\varphi_{n}^{*}\right) \nabla \phi_{n} \cdot\left(U_{n} \nabla \varphi_{n+1}+\nabla \varphi_{n}\right) \\
-\nabla \phi_{n} \cdot\left(U_{n}^{*} \nabla \varphi_{n+1}^{*}+\nabla \varphi_{n}^{*}\right)\left(U_{n} \varphi_{n+1}-\varphi_{n}\right)
\end{array},\right\}
\end{aligned}
$$

which leads to the equation of motion

$$
\begin{aligned}
& \frac{U_{n} \varphi_{n+1}-2 \varphi_{n}+U_{n-1}^{*} \varphi_{n-1}}{T^{2}}+\frac{\mu^{2}\left(U_{n} \varphi_{n+1}+4 \varphi_{n}+U_{n-1}^{*} \varphi_{n-1}\right)}{6} \\
& -\nabla \cdot\left[\frac{\left.\nabla \varphi_{n+1} U_{n}+4 \nabla \varphi_{n}+U_{n-1}^{*} \nabla \varphi_{n-1}\right]}{6}\right]= \\
& \frac{q^{2} T^{2}}{30}\left\{\left(\nabla \phi_{n}\right)^{2}\left[U_{n} \varphi_{n+1}-\varphi_{n}\right]-\left(\nabla \phi_{n-1}\right)^{2}\left[\varphi_{n}-U_{n-1}^{*} \varphi_{n-1}\right]\right\} \\
& -\frac{i q T}{12}\left\{\nabla \phi_{n} \cdot\left(U_{n} \nabla \varphi_{n+1}+\nabla \varphi_{n}\right)-\nabla \phi_{n-1} \cdot\left(\nabla \varphi_{n}+U_{n-1}^{*} \nabla \varphi_{n-1}\right)\right\} \\
& +\frac{i q T}{12} \nabla \cdot\left\{\nabla \phi_{n}\left(U_{n} \varphi_{n+1}-\varphi_{n}\right)+\nabla \phi_{n-1}\left(\varphi_{n}-U_{n-1}^{*} \varphi_{n-1}\right)\right\} .
\end{aligned}
$$

In the limit $T \rightarrow 0$ we recover the usual equation

$$
\left(\partial_{t}+i q \phi\right)\left(\partial_{t}+i q \phi\right) \varphi+\left(\mu^{2}-\nabla^{2}\right) \varphi \underset{c}{=} 0 .
$$

\section{The Dirac equation}

We turn now to the continuous time Dirac equation

$$
i \dot{\psi}=\underset{c}{=}(-i \boldsymbol{\alpha} \cdot \vec{\nabla}+\beta m) \psi
$$

which is obtained from the Lagrange density

$$
\mathcal{L}=\frac{1}{2} \psi^{+}[i \dot{\psi}+i \boldsymbol{\alpha} \cdot \vec{\nabla} \psi]+\frac{1}{2}\left[-i \dot{\psi}^{+}-i \psi^{+} \boldsymbol{\alpha} \cdot \overleftarrow{\nabla}\right] \psi-m \psi^{+} \beta \psi
$$

The virtual paths are taken to be

$$
\tilde{\psi}=\lambda \psi_{n+1}+\bar{\lambda} \psi_{n}, \quad \tilde{\psi}^{+}=\lambda \psi_{n+1}^{+}+\bar{\lambda} \psi_{n}^{+}
$$

and then we find the system function density

$$
\begin{aligned}
\mathcal{F}^{n}= & \frac{i}{2}\left\{\psi_{n}^{+} \psi_{n+1}-\psi_{n+1}^{+} \psi_{n}\right\} \\
& -\frac{T}{6}\left\{2 \psi_{n+1}^{+} \overleftrightarrow{D} \psi_{n+1}+\psi_{n+1}^{+} \overleftrightarrow{D} \psi_{n}+\psi_{n}^{+} \overleftrightarrow{D} \psi_{n+1}+2 \psi_{n}^{+} \overleftrightarrow{D} \psi_{n}\right\}
\end{aligned}
$$

where $\overleftrightarrow{D} \equiv-\frac{1}{2} i \boldsymbol{\alpha} \cdot \overleftrightarrow{\nabla}+\beta m$. From this we find the Cadzow equation of motion

$$
i \frac{\left(\psi_{n+1}-\psi_{n-1}\right)}{2 T}=\overrightarrow{H_{D}} \frac{\left(\psi_{n+1}+4 \psi_{n}+\psi_{n-1}\right)}{6},
$$


where $\overrightarrow{H_{D}} \equiv-i \boldsymbol{\alpha} \cdot \vec{\nabla}+\beta m$ and similarly for the conjugate field $\psi_{n}^{+}$. This is the required discretisation of the Dirac equation (127).

This equation has the same structure as that for the Grassmannian oscillator discussed in Paper I. To determine the regime for elliptic (particle like) behaviour, we first Fourier transform (131), defining

$$
\int d^{3} \mathbf{x e}^{-i \mathbf{p} \cdot \mathbf{x}} \psi_{n}(\mathbf{x})=\tilde{\psi}_{n}(\mathbf{p})
$$

and then (131) becomes

$$
(-3 i+D T) \tilde{\psi}_{n+1}+(3 i+D T) \tilde{\psi}_{n-1}=-4 D T \tilde{\psi}_{n}
$$

where $D \equiv \boldsymbol{\alpha} \cdot \mathbf{p}+\beta m$, which has the property

$$
D D \equiv \mathbf{p} \cdot \mathbf{p}+m^{2}=E^{2}
$$

$E$ being the definition of the particle's energy (keeping in mind there is no 'Hamiltonian' in this theory).

Now define the Dirac matrix

$$
\nu \equiv \frac{3 i D T+E^{2} T^{2}}{\sqrt{9 E^{2} T^{2}+E^{4} T^{4}}}
$$

and rescale the momentum fields via the rule

$$
\tilde{\psi}_{n} \equiv \nu^{n} \phi_{n}
$$

Then we find

$$
\phi_{n+1}+\phi_{n-1}=2 \eta \phi_{n}
$$

where $\eta=\frac{-2 E T}{\sqrt{9+E^{2} T^{2}}}$, which proves that oscillator behaviour is inherent in this system. Particle like solutions will occur for $\eta^{2}<1$, from which we deduce the condition $E T<\sqrt{3}$. This upper limit is one half of the upper limit (69) established for the discretised Klein-Gordon equation. Exactly the same phenomenon occurs in the Grassmannian oscillator studied in Paper $I$.

We shall leave the construction of conserved quantities such as the linear momentum and the Logan invariant built up from the ladder operators $a(\mathbf{p}), b(\mathbf{p})$, etc., as exercises. However, we will discuss here the charge and charge current densities.

First, we rewrite the Cadzow equations of motion (131) in the more useful form

$$
\begin{aligned}
& \left(-3 i+T \overrightarrow{H_{D}}\right) \psi_{n+1}=-4 T \overrightarrow{H_{D}} \psi_{n}-\left(3 i+T \overrightarrow{H_{D}}\right) \psi_{n-1} \\
& \psi_{n+1}^{+}\left(3 i+\overleftarrow{H_{D}} T\right)=-4 \psi_{n}^{+} \overleftarrow{H_{D}} T-\psi_{n-1}^{+}\left(-3 i+\overleftarrow{H_{D}} T\right)
\end{aligned}
$$

where $\overrightarrow{H_{D}} \equiv-i \boldsymbol{\alpha} \cdot \vec{\nabla}+\beta m, \overleftarrow{H_{D}} \equiv i \boldsymbol{\alpha} \cdot \overleftarrow{\nabla}+\beta m$

Now the system function (130) is invariant to the infinitesimal global gauge transformation

$$
\begin{aligned}
\psi_{n} & \rightarrow e^{i \theta} \psi_{n}, \quad \psi_{n}^{+} \rightarrow \psi_{n}^{+} e^{-i \theta} \\
\delta \psi_{n} & =i \theta \psi_{n}, \quad \delta \psi^{+}=-i \theta \psi_{n}^{+}
\end{aligned}
$$


so that there is a conserved charge given by

$$
Q^{n} \equiv \int d^{3} \mathbf{x}\left\{\psi_{n}^{+}\left(\frac{1}{2}+\frac{i T}{6} \overrightarrow{H_{D}}\right) \psi_{n+1}+\psi_{n+1}^{+}\left(\frac{1}{2}-\overleftarrow{H_{D}} \frac{T}{6}\right) \psi_{n}\right\}
$$

It is easy to use the equations of motion (138) to show that

$$
Q^{n} \underset{c}{=} Q^{n-1}
$$

The charge and current densities are then given by

$$
\begin{aligned}
\rho_{n} & =\psi_{n}^{+}\left(\frac{1}{2}+\frac{i T}{6} \overrightarrow{H_{D}}\right) \psi_{n+1}+\psi_{n+1}^{+}\left(\frac{1}{2}-\overleftarrow{H_{D}} \frac{i T}{6}\right) \psi_{n} \\
\mathbf{j}_{n} & =\frac{1}{6}\left\{\psi_{n}^{+} \boldsymbol{\alpha} \psi_{n-1}+4 \psi_{n}^{+} \boldsymbol{\alpha} \psi_{n}+\psi_{n-1}^{+} \boldsymbol{\alpha} \psi_{n}\right\}
\end{aligned}
$$

These collapse to the standard densities in the limit $T \rightarrow 0$, and satisfy the discrete time equation of continuity

$$
\frac{\rho_{n}-\rho_{n-1}}{T}+\nabla \cdot \mathbf{j}_{n}=0
$$

as required.

This completes our discussion of the free Dirac equation. The coupling of the Dirac field to the electromagnetic field in a gauge invariant way follows exactly the same procedure as with the charged Klein-Gordon field discussed in $\S 8$, with virtual paths following the rules given by (114). Second quantisation of the free Dirac field and QED will be discussed in the fourth in this series.

\section{Conclusions}

In this paper we have extended the methods outlined in the first paper of this series to classical fields, including scalar, vector, and spinor fields. The construction of equations of motion and invariants of the motion is straightforward and gauge invariance also can be readily built into the system function.

It is clear that the discretisation process does separate out timelike and spacelike terms in most equations, so that some of the elegance of a fully Lorentz covariant theory is lost. This is an inevitability in any discretisation process. However, this

disadvantage should be viewed alongside the potential advantages, which may be considerable.

First, our approach is exact, in that we do not countenance approximations within the theory itself, only in applications. Equations of motion are derived exactly from well-specified system functions, and invariants of the motion are precisely that, i.e., exact invariants of the motion and not approximate invariants of the motion. Next, there is a natural scale introduced into the theory, namely the fundamental time interval $T$. This influences the dynamics of the fields and leads to an upper limit for particle state energies, where the definition of particle state requires field amplitudes to remain bounded in time (corresponding to the condition $\eta^{2}<1$ in the harmonic 
oscillator). This holds some promise as a regulariser in quantum field theory, where in conventional calculations ad hoc cutoffs have to be introduced routinely.

One of the surprises discrete time mechanics has sprung is how well it can work. Although it is certainly not equivalent to continuous time mechanics, in almost every aspect of the latter the former can make a comparable statement. We do not have a Hamiltonian in discrete time mechanics, but we do have a system function which can be used to generate equations of the motion, invariants of the motion, and define quantisation, as discussed in Paper $I$. In the case of free fields we can readily construct a Logan invariant which substitutes for the Hamiltonian in a particularly useful way, closed allied with the particle content of the theory.

This raises the important question: if indeed all continuous time dynamical theories can be simulated by discrete time analogues, given a small enough $T$, so that no empirical test could distinguish between their predictions, what would force us to pick one approach rather than the other? One theory is elegant but badly defined mathematically in many places, whilst the other looks clumsier but is perhaps better defined mathematically.

Ultimately it may come down to a question of personal choice, which will be influenced by some intrinsic features of the approach. For instance, the construction of Feynman path integrals as infinite products of integrals cannot be carried out in the usual way in discrete time field theory, simply because in that theory there is no meaning to the taking of the limit $T \rightarrow 0$, other than to show some sort of consistency with conventional theory. Therefore, one of the serious objections to the conventional path integral formalism, that of being ill-defined, is greatly moderated in discrete time theory. There is something like a path integral, but it involves a finite number of time steps between initial and final times, as discussed in Paper $I$. In Paper III of this series we shall discuss the second quantisation of field theories using this approach.

\section{Acknowledgements}

We are pleased to thank Prof. J. Lukierski for his invaluable comments at an early stage in this work and for material assistance in finding important references and opportunities to discuss discrete time mechanics. 


\section{References}

[1] Jaroszkiewicz G \& Norton K, Principles of Discrete Time Mechanics: I. Particle Systems, (1996), accepted for publication in J. Phys. A: Math. Gen.

[2] Klimek K, Extension of q-deformed Analysis and q-deformed Models of Classical Mechanics, J. Phys. A: Math. Gen. 26, 955-967 (1993)

[3] Yamamoto H, Hayashi A, Hashimoto T \& Horibe M, Conserved Quantities of Field Theory on Discrete Spacetime, Prog. Theor. Phys. Vol. 93, No 1, 173-184 (1995)

[4] Hashimoto T, Hayashi A, Horibe M \& Yamamoto H, How to Quantize Fields Canonically on Discrete Space-Time, Prog. Theor. Phys., Vol. 94, No 2, 249-261 (1995)

[5] Cadzow J A, Discrete Calculus of Variations, Int. J. Control, vol 11, No 3, 393-407 (1970)

[6] Maeda S, Extension of Discrete Noether Theorem, Math. Japonica 26, no 1, 85-90 (1981), and references therein.

[7] Logan J D, First Integrals in the Discrete Variational Calculus, Aequat. Math. $\mathbf{9}, 210-220(1973)$

[8] Lukierski J, Nowicki A \& Ruegg H, New Quantum Poincaré Algebra and $\kappa$-deformed Field Theory, Phys. Lett. B293, 344 (1992) 